This manuscript has been submitted for publication in Geophysical Journal International.

Please note that, the manuscript has not undergone peer-review. Subsequent versions of this manuscript may have slightly different content. If accepted, the final version of this manuscript will be available via the 'Peer-reviewed Publication DOl' link on the right-hand side of this webpage. Please feel free to contact any of the authors. We welcome feedback! 


\title{
Simultaneous Inference of Plate Boundary Stresses and Mantle Rheology Using Adjoints: Large-Scale Two-Dimensional
} Models

\author{
Johann Rudi ${ }^{1 \star}$, Michael Gurnis ${ }^{2}$, and Georg Stadler ${ }^{3}$ \\ ${ }^{1}$ Mathematics and Computer Science Division, Argonne National Laboratory, Lemont, IL 60439, USA \\ ${ }^{2}$ Seismological Laboratory, California Institute of Technology, Pasadena, CA 91125, USA \\ ${ }^{3}$ Courant Institute of Mathematical Sciences, New York University, New York, NY 10012, USA
}

7 November 2021

\section{SUMMARY}

Plate motions are a primary surface constraint on plate and mantle dynamics and rheology, plate boundary stresses, and the occurrence of great earthquakes. Within an optimization method, we use plate motion data to better constrain uncertain mantle parameters. For the optimization problem characterizing the maximum a posteriori rheological parameters we derive gradients using adjoints and expressions to approximate the posterior distributions for stresses within plate boundaries. We apply these methods to a 2-D cross section from the western to eastern Pacific, with temperature distributions and fault zone geometries developed primarily from seismic and plate motion data. We find that the best-fitting stress exponent, $n$, is about 2.8 and the yield stress about $100 \mathrm{MPa}$ or less. The normal stress on the interplate fault zones is about $100 \mathrm{MPa}$ and the shear stresses about $10 \mathrm{MPa}$ or less.

Key words: Plate driving forces, mantle convection, geophysical inference

\footnotetext{
* Email: jrudi@anl.gov
} 


\section{INTRODUCTION}

Plate motion is the result of a force balance involving mantle and lithosphere rheology, the longterm strength of fault zones that define plate boundaries, and buoyancy forces. But considerable disagreement and uncertainty about these characteristics remain, primarily because of the complexity of mechanical properties. The creep strength of the mantle is highly nonlinear and is dependent on pressure, temperature, and composition (Ranalli, 1995), while a range of strain- and composition-dependent processes control the development and evolution of fault zones (Scholz, 1990), such that mantle downwellings are characterized by relatively strong slabs that slide by the overriding plate. Plate bending (Buffett, 2006) and interplate coupling (Scholz \& Campos, 2012) are distinct processes, each constrained observationally. These processes need to be captured in models in order to decipher the plate tectonic controls on interplate coupling and how slab strength (Billen, 2008) and mantle rheology balance to control plate motions and mantle flow.

In two-dimensional forward models (i.e., computations with assumed parameters), the treatment of constitutive relations for creep and faults (shear zones) approximately consistent with those suggested from laboratory work has been accomplished in cross-sectional models of subduction (Zhong \& Gurnis, 1995; Zhong et al., 1998; Billen \& Hirth, 2007; Crameri et al., 2012; Gerya, 2011; Gérault et al., 2015; Garel et al., 2014). Indeed, assuming strain rate- and temperaturedependent viscosities has led to forward models that are consistent with generic subduction zone evolution (Zhong \& Gurnis, 1995; Garel et al., 2014; Burkett \& Billen, 2009). In global models,

20 these constitutive relationships and parameterizations are often simplified (Conrad \& LithgowBertelloni, 2002) so as to make the problem computationally tractable. The simplifications entail the use of linear viscosities, potentially smaller lateral variations in viscosity that could exist (as suggested by 2-D models), and are usually low resolution. In subduction models, low resolution is limiting because it changes the character of deformation and flow within a subduction zone: Instead of a relatively strong plate that slides by and is resisted by the overriding plate, the otherwise strong plate advects through a wide, low-viscosity zone such that the plate neither bends nor is strong. These limitations have been partially overcome in models with resolutions of up to $1 \mathrm{~km}$ within and near the shear zones defining subducting plate boundaries (Stadler et al., 2010, 
Alisic et al. 2012, 2010). But such models also have their limitations, especially in that they are

so forward computations that only reasonably fit plate motions. Such models show that the degree of fault coupling likely varies between subduction zones, but the computational cost of each forward model precludes finding the true best-fitting model as well as the correlation between parameters.

To accurately estimate the forces controlling plate motions, we need an optimization scheme that faithfully resolves the key mechanical, rheological, and geometric aspects of the system. Here we move in that direction by expanding on the adjoint approach to efficiently compute derivatives (Ratnaswamy et al., 2015) with actual plate motions and seismic constraints in 2-D cross-sectional models. Besides applying the method to geophysical data and inferring mantle properties, we enhance the method by inferring the stress within plate margins. We determine the conditional distributions of these stresses and inferred rheological parameters. Moreover, we demonstrate the stability of the numerical methods for inference and show that we can constrain the nonlinear exponent of mantle deformation, the activation energy, the prefactor to a rheological model, and the effective viscosity and stress within fault zones, as well as the trade-offs among all of these quantities. In essence, we refine optimization methods for geodynamic models using 2-D models, paving the way for the geodynamic inverse models that will require computation of the entire spherical problem with global temperature and plate motion constraints.

\section{GOVERNING EQUATIONS: THE FORWARD PROBLEM}

The underlying physics of mantle flow is governed by the creeping flow of mantle rocks over geological time scales. Over time scales greater than thousands of years, deformation of the mantle behaves as a viscous fluid governed by the conservation of mass and momentum, which can be 5o modeled by the incompressible Stokes equations

$$
\begin{array}{ll}
-\nabla \cdot \boldsymbol{u}=0 & \text { in } \Omega, \\
-\nabla \cdot \boldsymbol{\sigma}=-\mathrm{RaTe}_{r} & \text { in } \Omega,
\end{array}
$$


where $\Omega \subset \mathbb{R}^{n}, n=2,3$ is a bounded two- or three-dimensional domain with boundary $\partial \Omega$. On that boundary we assume no-outflow and tangential free-slip boundary conditions,

$$
\boldsymbol{u} \cdot \boldsymbol{n}=0, \quad \boldsymbol{T}(\boldsymbol{\sigma n})=0 \quad \text { on } \partial \Omega .
$$

Here $\boldsymbol{u}: \Omega \rightarrow \mathbb{R}^{n}$ and $p: \Omega \rightarrow \mathbb{R}$ are the unknown velocity and the pressure, respectively. The viscous stress tensor is defined as $\boldsymbol{\sigma}=2 \eta\left(\boldsymbol{x}, T, \dot{\varepsilon}_{\mathrm{II}}, \boldsymbol{m}\right) \dot{\boldsymbol{\varepsilon}}-p \mathbf{I}$, where $\dot{\boldsymbol{\varepsilon}}=\frac{1}{2}\left(\nabla \boldsymbol{u}+\nabla \boldsymbol{u}^{T}\right)$ is the strain rate tensor. Moreover, $\eta=\eta\left(\boldsymbol{x}, T, \dot{\varepsilon}_{\mathrm{II}}, \boldsymbol{m}\right)$ is the (effective) viscosity, which depends on the location in the domain $\boldsymbol{x}$, a given temperature field $T$, on (the square root of) the second invariant of the strain rate tensor $\dot{\varepsilon}_{\mathrm{II}}=\left(\frac{1}{2} \dot{\varepsilon}: \dot{\varepsilon}\right)^{1 / 2}$, and on rheology parameters summarized in the vector $\boldsymbol{m}$, to be specified later. The momentum equation (2) is driven by thermal buoyancy, where Ra is the thermal Rayleigh number and $\boldsymbol{e}_{r}$ is the negative unit vector in the direction of increasing gravity. In (3), $\boldsymbol{n}$ represents the outward unit normal vector at the boundary $\partial \Omega$, and $\boldsymbol{T}=\mathbf{I}-\boldsymbol{n} \otimes \boldsymbol{n}$ is the tangential projection operator, where $\otimes$ denotes the outer product.

A nonlinear rheology is used in the upper mantle to account for dislocation creep and a linear rheology in the lower mantle for diffusion creep. A global yield stress $\sigma_{y}$ is used that allows for the dynamic weakening, which is triggered primarily within the hinge zones as slabs bend when entering the mantle. At a point $\boldsymbol{x} \in \Omega$, the viscosity is described as

$$
\eta\left(\boldsymbol{x}, T, \dot{\varepsilon}_{\mathrm{II}}, \boldsymbol{m}\right)=\eta_{\min }+\min \left\{\frac{\sigma_{y}}{2 \dot{\varepsilon}_{\mathrm{II}}}, \omega(\boldsymbol{x}) \min \left\{a(T) \dot{\varepsilon}_{\mathrm{II}}^{\frac{1-n}{n}}, \eta_{\max }\right\}\right\}
$$

where $\eta_{\min }$ and $\eta_{\max }$ are the minimum and maximum bounds of the viscosity, respectively; $\omega(\boldsymbol{x})$ is the function describing the reduction of viscosity for each weak zone defining subducting plate boundaries; and $a(T)$ is the temperature-dependent component of viscosity. Further, $\sigma_{y}$ is a yield stress triggering plastic failure. We denote $n$ as the stress exponent causing strain rate weakening when $n>1$, and we call its inverse $1 / n$ the strain rate weakening exponent. As detailed in the next section, the vector $\boldsymbol{m}$ collects the parameters we aim to infer from data, such as the strain rate exponent, the weak zone factors, and the yield stress, possibly after some transformation.

The normal Arrhenius equation is linearized as the adjusted Frank-Kamenetskii law (Frank- 
Kameneëtìskiæi, 1969)

$$
a(T)=\eta_{0} \exp (\beta(0.5-T) / n),
$$

where $\beta$ is the nondimensional activation energy and $\eta_{0}$ is the viscosity prefactor. The reduction in viscosity that represents the fault zones between converging plates at subduction zones is described by using the weakening function

$$
\omega(\boldsymbol{x})=\prod_{i} 1-\left(1-w_{i}\right) \chi_{i}(\boldsymbol{x}), \quad \text { where } \quad \chi_{i}(\boldsymbol{x})=\exp \left(-\frac{\max \left\{0, \operatorname{dist}\left(\Gamma_{i}, \boldsymbol{x}\right)-d\right\}^{2}}{2 b^{2}}\right)
$$

with a weakening coefficient $0<w_{i} \leq 1$ that is, generally, different for each subduction zone with index $i$; $\operatorname{dist}\left(\Gamma_{i}, \boldsymbol{x}\right)$ denotes the minimal distance of $\boldsymbol{x}$ and the center surface describing the weak zone $\Gamma_{i}, d$ is the width of the zone of full weakening, and $b$ is the length-scale of smoothing. Although the parameters governing $\omega(\boldsymbol{x})$ are intrinsic parameters in the viscosity formulation (4), the viscosity within the weak zones is not solely intrinsic, because strain rate weakening plays an important role.

\section{ESTIMATING PARAMETERS: THE BAYESIAN INVERSE PROBLEM}

Next we summarize the Bayesian inference approach and the computation of derivatives using the adjoint method in Section 3.1. We describe the parameters and the effect of nonlinear transformations in Section 3.2. In Section 3.3 we show how Bayesian estimation of the rheology parameters can be used to estimate uncertain physics quantities such as the plate boundary shear and normal stresses.

\subsection{Bayesian inverse problem and computation of derivatives}

We now briefly summarize the Bayesian approach to parameter estimation. For a general introduction on the subject, we refer to Tarantola (2005) and Kaipio \& Somersalo (2005) and, in the context of mantle dynamics, to Ratnaswamy et al. (2015). We combine the uncertain rheology parameters that we aim to infer from observations into a parameter vector $\boldsymbol{m} \in \mathbb{R}^{p}$; the inference parameters are discussed in Section 3.2. In the current work, observational data are considered to be plate 
velocities at the top surface of $\Omega$, which we denote by $\partial \Omega_{\mathrm{obs}}$. Since plate movement is assumed to be rigid, one velocity vector (or Euler pole for spheres) is available for each plate. We interpolate these velocity vectors to obtain one tangential velocity field at the surface per plate. These velocity fields constitute the observational data of the inverse problem and are only considered sufficiently far away from nonrigid plate boundaries. Corresponding to the observational data, denoted as $\boldsymbol{d}_{\mathrm{obs}}$, we define the observations from the solution of the Stokes equations $(1-2)$ for given parameters $\boldsymbol{m}$ as $\boldsymbol{f}(\boldsymbol{m})$, which is supported on $\partial \Omega_{\mathrm{obs}}$; and we refer to $\boldsymbol{f}$ as the parameter-to-observable map. Moreover, we decompose $\boldsymbol{f}(\boldsymbol{m})$ into an observation operator $\boldsymbol{b}(\cdot)$ applied to the solution $\boldsymbol{u}(\boldsymbol{m})$ of the Stokes equations for given parameters $\boldsymbol{m}$; hence, $\boldsymbol{f}(\boldsymbol{m})=\boldsymbol{b}(\boldsymbol{u}(\boldsymbol{m}))$.

In a Bayesian inverse problem, the parameters $\boldsymbol{m}$ and data $\boldsymbol{d}_{\text {obs }}$ are considered random variables to model our imperfect knowledge about these parameters. Furthermore, we assume that the combined measurement and model error is additive and that the error $\left(\boldsymbol{d}_{\mathrm{obs}}-\boldsymbol{f}(\boldsymbol{m})\right)$ is Gaussian with zero mean and covariance matrix $\mathcal{C}_{\text {data }}$. We additionally choose a prior distribution for the parameters $\boldsymbol{m}$ that is Gaussian with mean $\boldsymbol{m}_{0}$ and covariance matrix $\mathcal{C}_{\text {prior }}$. With these assumptions, it follows that the posterior distribution, which is obtained as the solution of the Bayesian inverse problem, has the density

$$
\boldsymbol{\pi}_{\text {post }}(\boldsymbol{m}) \propto \exp (-\mathcal{J}(\boldsymbol{m}))
$$

where $\propto$ means equal up to a (normalization) constant that makes $\pi_{\text {post }}$ a proper density. The real-valued exponent $\mathcal{J}$ in the posterior density is defined as

$$
\mathcal{J}(\boldsymbol{m})=\frac{1}{2}\left(\boldsymbol{d}_{\mathrm{obs}}-\boldsymbol{f}(\boldsymbol{m})\right)^{T} \mathcal{C}_{\mathrm{data}}^{-1}\left(\boldsymbol{d}_{\mathrm{obs}}-\boldsymbol{f}(\boldsymbol{m})\right)+\frac{1}{2}\left(\boldsymbol{m}-\boldsymbol{m}_{0}\right)^{T} \mathcal{C}_{\text {prior }}^{-1}\left(\boldsymbol{m}-\boldsymbol{m}_{0}\right),
$$

where the first term is a cost measuring the misfit between data and model output $t^{\dagger}$ and the second term penalizes deviations of parameters from prior knowledge. Since within mapping $\boldsymbol{f}(\boldsymbol{m})$, the Stokes solution $(\boldsymbol{u}, p)$ depends nonlinearly on the parameters $\boldsymbol{m}$, the posterior distribution (7) is non-Gaussian, and thus its statistical characterization is challenging. Since we aim at problems where we infer multiple global rheology parameters as well as multiple plate boundary weak zone

$\dagger$ Note that in the case of observations being plate motions at the surface $\partial \Omega_{\mathrm{obs}}$, the evaluation of the cost for the data misfit entails computing an integral over $\partial \Omega_{\mathrm{obs}}$. In 8 the integral is implied in the quadratic form associated with the inverse error covariance matrix $\mathcal{C}_{\mathrm{data}}^{-1}$. 
factors, it is computationally difficult to fully explore the high-dimensional posterior distribution (e.g., through sampling methods), because of the computational cost that each solution of a highresolution nonlinear Stokes system entails. Thus we have to resort to approximations of $\pi_{\text {post }}$. One such approximation is to first minimize $\mathcal{J}(\boldsymbol{m})$ over $\boldsymbol{m}$. This yields the maximum a posteriori (MAP) point $\boldsymbol{m}_{\text {map }}$ and requires solving an optimization problem that is implicitly governed by the nonlinear Stokes equation. Next, an approximation of the posterior distribution is found by linearizing $\boldsymbol{f}$ about the MAP point. If we denote this linearization by $\boldsymbol{F}$, the resulting approximation of the posterior is Gaussian with mean $\boldsymbol{m}_{\text {map }}$ and covariance matrix $\left(\boldsymbol{F}^{T} \mathcal{C}_{\text {data }}^{-1} \boldsymbol{F}+\mathcal{C}_{\text {prior }}^{-1}\right)^{-1}$. With the MAP point and Gaussian approximation of (7), it now becomes computationally tractable to locally describe $\pi_{\text {post }}$.

The MAP point as well as the Gaussian approximation of the posterior requires derivatives of $\mathcal{J}$ with respect to $\boldsymbol{m} \in \mathbb{R}^{p}$. We utilize adjoint methods to compute these derivatives efficiently, because the computational cost of adjoint-based derivatives is independent of the number of parameters $p$. Next, we introduce adjoint variables obtained as solutions to adjoint equations derived from the Lagrangian (Hinze et al., 2009), which incorporate the minimization objective (8) and the Stokes equations $(1-2)$ in weak form. The Lagrangian function $\mathcal{L}$ is defined as

$$
\begin{aligned}
\mathcal{L}(\boldsymbol{u}, p, \boldsymbol{v}, q, \boldsymbol{m})=\tilde{\mathcal{J}}(\boldsymbol{m}, \boldsymbol{u}) & +\int_{\Omega} 2 \eta\left(\boldsymbol{x}, T, \dot{\varepsilon}_{\mathrm{II}}, \boldsymbol{m}\right) \dot{\boldsymbol{\varepsilon}}(\boldsymbol{u}): \dot{\boldsymbol{\varepsilon}}(\boldsymbol{v}) d \boldsymbol{x} \\
& -\int_{\Omega} p(\nabla \cdot \boldsymbol{v}) d \boldsymbol{x}-\int_{\Omega} q(\nabla \cdot \boldsymbol{u}) d \boldsymbol{x}+\int_{\Omega} \operatorname{Ra} T \mathbf{e}_{r} \cdot \boldsymbol{v} d \boldsymbol{x},
\end{aligned}
$$

where $\boldsymbol{v}$ and $q$ are the adjoint velocity and pressure and the arguments $\boldsymbol{m}, \boldsymbol{u}$ of $\tilde{\mathcal{J}}$ in $(9)$, are now considered independent variables; thus

$$
\tilde{\mathcal{J}}(\boldsymbol{m}, \boldsymbol{u})=\frac{1}{2}\left(\boldsymbol{d}_{\mathrm{obs}}-\boldsymbol{b}(\boldsymbol{u})\right)^{T} \mathcal{C}_{\mathrm{data}}^{-1}\left(\boldsymbol{d}_{\mathrm{obs}}-\boldsymbol{b}(\boldsymbol{u})\right)+\frac{1}{2}\left(\boldsymbol{m}-\boldsymbol{m}_{0}\right)^{T} \mathcal{C}_{\text {prior }}^{-1}\left(\boldsymbol{m}-\boldsymbol{m}_{0}\right)
$$

The gradient $\mathcal{G}(\boldsymbol{m})$ of $\mathcal{J}(\boldsymbol{u}(\boldsymbol{m}))$ in $(8)$ with respect to $\boldsymbol{m}$ is given by the gradient of $\mathcal{L}$ with respect to $\boldsymbol{m}$, provided that all variations of the Lagrangian with respect to $(\boldsymbol{v}, q)$ and $(\boldsymbol{u}, p)$ vanish; see Tröltzsch (2010); Borzì \& Schulz (2012). These conditions for stationarity of the Lagrangian imply 


$$
\begin{array}{ll}
-\nabla \cdot \boldsymbol{v}=0 & \text { in } \Omega \\
-\nabla \cdot \boldsymbol{\tau}=0 & \text { in } \Omega
\end{array}
$$

with the adjoint stress

$$
\boldsymbol{\tau}:=\left(2 \eta \mathbb{I}+\frac{\partial \eta}{\partial \dot{\varepsilon}_{\mathrm{II}}} \frac{\dot{\boldsymbol{\varepsilon}}(\boldsymbol{u}) \otimes \dot{\boldsymbol{\varepsilon}}(\boldsymbol{u})}{\dot{\varepsilon}_{\mathrm{II}}}\right) \dot{\varepsilon}(\boldsymbol{v})-q \mathbf{I}
$$

and with boundary conditions for $v$ as in (3) but with

$$
\boldsymbol{T}(\boldsymbol{\tau} \boldsymbol{n})=\left[\frac{d \boldsymbol{b}}{d \boldsymbol{u}}(\boldsymbol{u})\right]^{T} \mathcal{C}_{\mathrm{data}}^{-1}\left(\boldsymbol{d}_{\mathrm{obs}}-\boldsymbol{b}(\boldsymbol{u})\right) \quad \text { on } \partial \Omega_{\mathrm{obs}}
$$

This means that the difference between the observation data $\boldsymbol{d}_{\mathrm{obs}}$ and the model output $\boldsymbol{b}(\boldsymbol{u})$ drives the adjoint equation. Note that the adjoint stress $\tau$ defined in (13) depends on the Stokes solution $\boldsymbol{u}$ and that $\otimes$ denotes the outer product between second-order tensors. The gradient now can be evaluated as the sensitivity of $\mathcal{L}$ in $(9)$ with respect to $\boldsymbol{m}$,

$$
\mathcal{G}(\boldsymbol{m})=\mathcal{C}_{\text {prior }}^{-1}\left(\boldsymbol{m}-\boldsymbol{m}_{0}\right)+\int_{\Omega} 2 \frac{d \eta\left(\boldsymbol{x}, T, \dot{\varepsilon}_{\mathrm{II}}(\boldsymbol{u}), \boldsymbol{m}\right)}{d \boldsymbol{m}} \dot{\boldsymbol{\varepsilon}}(\boldsymbol{u}): \dot{\boldsymbol{\varepsilon}}(\boldsymbol{v}) d \boldsymbol{x},
$$

using the solutions $u$ and $v$ of the Stokes equations (11-2) and the adjoint Stokes equations $(11,12)$, respectively.

\subsection{Inference parameters and their transformations}

We collect the parameters we are inverting for in the parameter vector $\boldsymbol{m}$. In order to avoid difficulties that arise when physical parameters are scaled very differently or must satisfy sign conditions, the inference parameters in $\boldsymbol{m}$ are (nonlinear) transformations of these physical parameters. In particular, since the weak zone factors must satisfy $0<w_{i} \leq 1$, the corresponding inversion parameters are $m_{w_{i}} \propto \log \left(w_{i}\right)$. Since the nonlinear exponent $n$ is responsible for strain rate weakening via $\dot{\varepsilon}_{\mathrm{II}}^{\frac{1}{n}-1}$, we consider the inference parameter $m_{n} \propto 1 / n$; this choice is further discussed in Section 5 . Additionally, we scale all parameters to order 1 to avoid numerical difficulties in the optimization problem for the MAP estimate. Note that in a Bayesian approach, nonlinear parameter 
transformations affect the Bayesian posterior. First, transformations change the prior distribution, which is typically formulated in terms of the transformed parameters, that is, components of the vector $\boldsymbol{m}$. This has an effect on the solution of the optimization characterizing the MAP estimate. Additionally, nonlinear transformations of random variables lead to a transformation of the underlying density functions through multiplication with the determinant of the Jacobian of the transformation. Consequently, such nonlinear transformations have implications on the posterior distribution and its approximations. They are more than a mathematical tool to, for instance, enforce positivity of a physical parameter. Thus, when choosing parameters in a Bayesian inference problem, one should take these transformations into account and carefully consider the prior information one incorporates through transformations and assumptions on these transformed parameters. The next section discusses how the parameters $\boldsymbol{m}$ can be inferred, together with estimates of their uncertainty, from observation data.

\subsection{Uncertainty quantification for physical quantities}

Here we derive estimates for physical quantities of interest (QoIs) from a computed solution of the Bayesian inverse problem from Section 3.1. In addition, we are able to propagate uncertainties from parameter estimates in the rheological relationship to those physical quantities. Specifically, we focus on the average plate boundary shear stresses and normal tractions since these quantities are central to the linking of geodynamic to seismogeneic processes. For the $i$ th weak zone, the average normal traction $\bar{\sigma}_{i}^{(n)}$ and the average shear stress $\overline{\boldsymbol{\sigma}}_{i}^{(t)}$ (i.e., shear traction) are defined as

$$
\bar{\sigma}_{i}^{(n)}=\frac{1}{V_{\chi_{i}}} \int_{\Omega} \chi_{i} \boldsymbol{n}_{w} \cdot\left(\boldsymbol{\sigma} \boldsymbol{n}_{w}\right) d \boldsymbol{x}, \quad \overline{\boldsymbol{\sigma}}_{i}^{(t)}=\frac{1}{V_{\chi_{i}}} \int_{\Omega} \chi_{i}\left(\mathbf{I}-\boldsymbol{n}_{w} \otimes \boldsymbol{n}_{w}\right)\left(\boldsymbol{\sigma} \boldsymbol{n}_{w}\right) d \boldsymbol{x},
$$

where $V_{\chi_{i}}=\int_{\Omega} \chi_{i} d \boldsymbol{x}$ is the volume of the weak zone $i$ and $\chi_{i}(\boldsymbol{x}) \in\{0,1\}$ equals one inside and equals zero outside of the weak zone. Moreover, $\boldsymbol{n}_{w}=\boldsymbol{n}_{w}(\boldsymbol{x})$ is the unit normal vector on the weak zone's center surface $\Gamma_{i}$, evaluated at the point on $\Gamma_{i}$ that is nearest to $\boldsymbol{x}$.

The normal and shear components of the stress are important because they effectively give the resisting stresses along the plate boundaries. The larger the resisting stress, the more mechanically coupled a plate boundary, and vice versa. Ultimately, these quantities will allow us to establish 
a relationship between seismic and mechanical coupling by comparing the weakening factors $w_{i}$ with the geographic variability of great earthquake occurrence and characteristics.

Our goal is to estimate the distribution of the quantities $[16$ given estimates and uncertainties of the rheological parameters $\boldsymbol{m}$. For that purpose, let us summarize the quantities we are interested in, $\bar{\sigma}_{i}^{(n)}$ and $\overline{\boldsymbol{\sigma}}_{i}^{(t)}$, in a vector $\boldsymbol{q}$. We consider $\boldsymbol{q}$ as a function of the velocity $\boldsymbol{u}$ and, in general, also as a function of the uncertain parameters $\boldsymbol{m}$; hence

$$
\boldsymbol{q}=\boldsymbol{q}(\boldsymbol{u}(\boldsymbol{m}), \boldsymbol{m})
$$

A Taylor expansion of $\boldsymbol{q}$ with respect to $\boldsymbol{m}$ about the MAP point $\boldsymbol{m}_{\text {map }}$ yields

$$
\boldsymbol{q}(\boldsymbol{m}) \approx \boldsymbol{q}\left(\boldsymbol{m}_{\mathrm{map}}\right)+\boldsymbol{J}\left(\boldsymbol{m}-\boldsymbol{m}_{\mathrm{map}}\right)
$$

where $\boldsymbol{J}$ denotes the Jacobian of $\boldsymbol{q}(\boldsymbol{m})$ at the MAP point,

$$
\boldsymbol{J}=\frac{d \boldsymbol{q}}{d \boldsymbol{m}}\left(\boldsymbol{m}_{\mathrm{map}}\right)=\left.\left[\frac{\partial \boldsymbol{q}}{\partial m_{1}}, \ldots, \frac{\partial \boldsymbol{q}}{\partial m_{p}}\right]\right|_{\boldsymbol{m}=\boldsymbol{m}_{\mathrm{map}}} \text { with } \frac{\partial \boldsymbol{q}}{\partial m_{j}}=\left[\frac{\partial q_{1}}{\partial m_{j}}, \ldots, \frac{\partial q_{i}}{\partial m_{j}}, \ldots\right]^{T}
$$

For the next step, we assume to have computed the Gaussian approximation of the posterior distribution for $\boldsymbol{m}$ (see Section 3.1) with mean $\boldsymbol{m}_{\text {map }}$ and the covariance matrix $\mathcal{C}_{\text {post }}$. By utilizing this Gaussian posterior and the truncated Taylor series $(18)$, we arrive at an approximation for the resulting distribution of $\boldsymbol{q}$. It has the mean $\boldsymbol{q}_{\text {map }}=\boldsymbol{q}\left(\boldsymbol{m}_{\text {map }}\right)$ and the covariance matrix $\mathcal{C}_{\mathrm{QoI}}=\boldsymbol{J} \mathcal{C}_{\text {post }} \boldsymbol{J}^{T}$. This follows from $(18)$ and basic properties of affine-transformed Gaussian random variables. While $\boldsymbol{q}_{\text {map }}$ can be evaluated directly, the covariance $\mathcal{C}_{\text {QoI }}$ requires the Jacobian (19), which entails the sensitivity vectors $\partial \boldsymbol{q} / \partial m_{j}$. Note that $\boldsymbol{q}$ in (17) depends on $\boldsymbol{m}$ through the solution $\boldsymbol{u}$ of the Stokes equations.

One straightforward way of computing the Jacobian (19) approximately is to use forward sensitivities. It is carried out with finite differences, where each parameter $m_{i}$ is perturbed by a small value $\delta$ and the QoI is evaluated at the perturbed parameter,

$$
\boldsymbol{J} \boldsymbol{e}_{i} \approx \frac{\boldsymbol{q}\left(\boldsymbol{m}_{\text {map }}\right)-\boldsymbol{q}\left(\boldsymbol{m}_{\mathrm{map}}+\delta \boldsymbol{e}_{i}\right)}{\delta}
$$


where $\boldsymbol{e}_{i}$ is a vector with entry one at index $i$ and zero otherwise. In order to compute all entries of $\boldsymbol{J}$, the number of nonlinear Stokes solves amounts to the product of the number of QoIs and parameter dimension $p$. We are using forward sensitivities to compute the small amount of six QoIs and have a parameter dimension of eight. Therefore, the postprocessing step of propagating uncertainties to QoIs is computationally feasible for the inverse problem we are targeting.

Remark 3.1. (Adjoint sensitivities) An alternative way of computing (19) would be using adjoints in a similar way as in Section 3.1 . This has the advantage of the computational cost being independent of parameter dimension $p$; however, it requires additional implementation efforts for new differential operators. We present the derivations here for completeness, but we used the finite difference technique to approximate (19) in our numerical experiments.

To derive the sensitivities in (19), we use a formal Lagrangian procedure analogously to the approach in Section 3.1. Specifically, in Equation 9, we substitute $\mathcal{J}(\boldsymbol{m}, \boldsymbol{u})$ by one scalar-valued QoI $q_{i}(\boldsymbol{m}, \boldsymbol{u})$ (i.e., the $i$ th component of $\boldsymbol{q}$ ) and carry out the same formal Lagrange procedure as in Section 3.1. Since we performed the substitution of $\mathcal{J}(\boldsymbol{m}, \boldsymbol{u})$ with $q_{i}(\boldsymbol{m}, \boldsymbol{u})$, the right-hand side of the adjoint equation (12) becomes the derivative of $q_{i}(\boldsymbol{m}, \boldsymbol{u})$ with respect to $\boldsymbol{u}$. Therefore, for every component $q_{i}$ of the QoI vector $\boldsymbol{q}$, an adjoint equation with a different right-hand side must be solved. In addition to the modified adjoint equation, a new term appears in the gradient expression (15), which is the derivative of the QoI's Lagrangian with respect to the parameters $\boldsymbol{m}$. This is due to the explicit dependence of $q_{i}(\boldsymbol{m}, \boldsymbol{u})$ on the parameters $\boldsymbol{m}$.

We consider the QoI to be the normal traction $\bar{\sigma}_{i}^{(n)}$ in the $i$ th weak zone. The derivative of $\bar{\sigma}_{i}^{(n)}$ with respect to $\boldsymbol{u}$, given an arbitrary direction $\boldsymbol{v}$, is

$$
\frac{d \bar{\sigma}_{i}^{(n)}}{d \boldsymbol{u}}(\boldsymbol{v})=\frac{1}{V_{\chi_{i}}} \int_{\Omega} \chi_{i}(\boldsymbol{x}) \boldsymbol{n}_{w} \cdot(\boldsymbol{\tau}+q \mathbf{I}) \boldsymbol{n}_{w} d \boldsymbol{x}
$$

where the adjoint stress $\tau$ has been defined in (13). The adjoint equations for the QoI $\bar{\sigma}_{i}^{(n)}$, therefore, have the negative of (21) in place of the right-hand side in (12). The sensitivities of the average shear stress $\overline{\boldsymbol{\sigma}}_{i}^{(t)}$ with respect to $\boldsymbol{u}$ are obtained similarly to the procedure that resulted in (21). 


\section{MODEL SETUP}

We have constructed a set of model constraints based on global observations with the components: a global temperature distribution, the geometry of faults, and the kinematics of plate motion. The temperature model has been constructed globally in a spherical shell, from which selected cross sections are taken. The temperature of the oceanic lithosphere follows a half-space cooling model using updates to the digital grid of the age of the oceanic plates (Müller et al., 1997). A thermal age was used within continents divided into three regions: cratons (300 Ma), areas near subduction zones (75 Ma), and other areas (200 Ma), as detailed in Stadler et al. (2010). The thermal structure of slabs was constructed as follows. Initially the top surface of the slabs was derived from the Slabs 1.0 surface, based on detailed seismic constraints, including seismicity and seismic reflection profiles (Hayes et al., 2012). With normals pointing downward from this surface, an initial thermal structure of slabs based on the half-space model using the age of the plate at the position of the trench was generated. This procedure ensured continuity of the thermal structure of the oceanic lithosphere. Then, thermal conduction was computed at each depth over a duration equal to the travel time to reach the depth with the local convergence velocity (using the relative velocity vector). Although solved only with conduction, this procedure resulted in thermal structures close to those obtained in fully dynamic models. The tops of thermal slabs were sharp in the corner of the mantle wedge and then progressively became diffusive with depth. Within the lower mantle, the thermal structure was based on a scaled seismic tomographic model, from the $\mathrm{S}$ waves in a joint $\mathrm{P}$ and $\mathrm{S}$ wave inference (Simmons et al., 2012). The lithosphere and upper mantle models and the upper and lower mantles were blended together at $75 \mathrm{~km}$ and $550 \mathrm{~km}$ depths, respectively, as shown in cross section in Fig. 1. We have used the seismotectonic approach for the shallower mantle and tomographic approach for the deeper mantle, since the seismic tomography models for slabs tend to be spatially irregular. Such irregular structure is generally not consistent with Benioff zone seismicity.

On the surface, a velocity field from MORVEL56 (Argus et al., 2011) in a no-net-rotation (NNR) reference frame was used. The main cross section defines a great circle arc, with local unit vector $\mathbf{d}$ in the direction of the circle and with velocity $v_{x s}=\mathbf{d} \cdot \mathbf{v}$. The NNR reference 

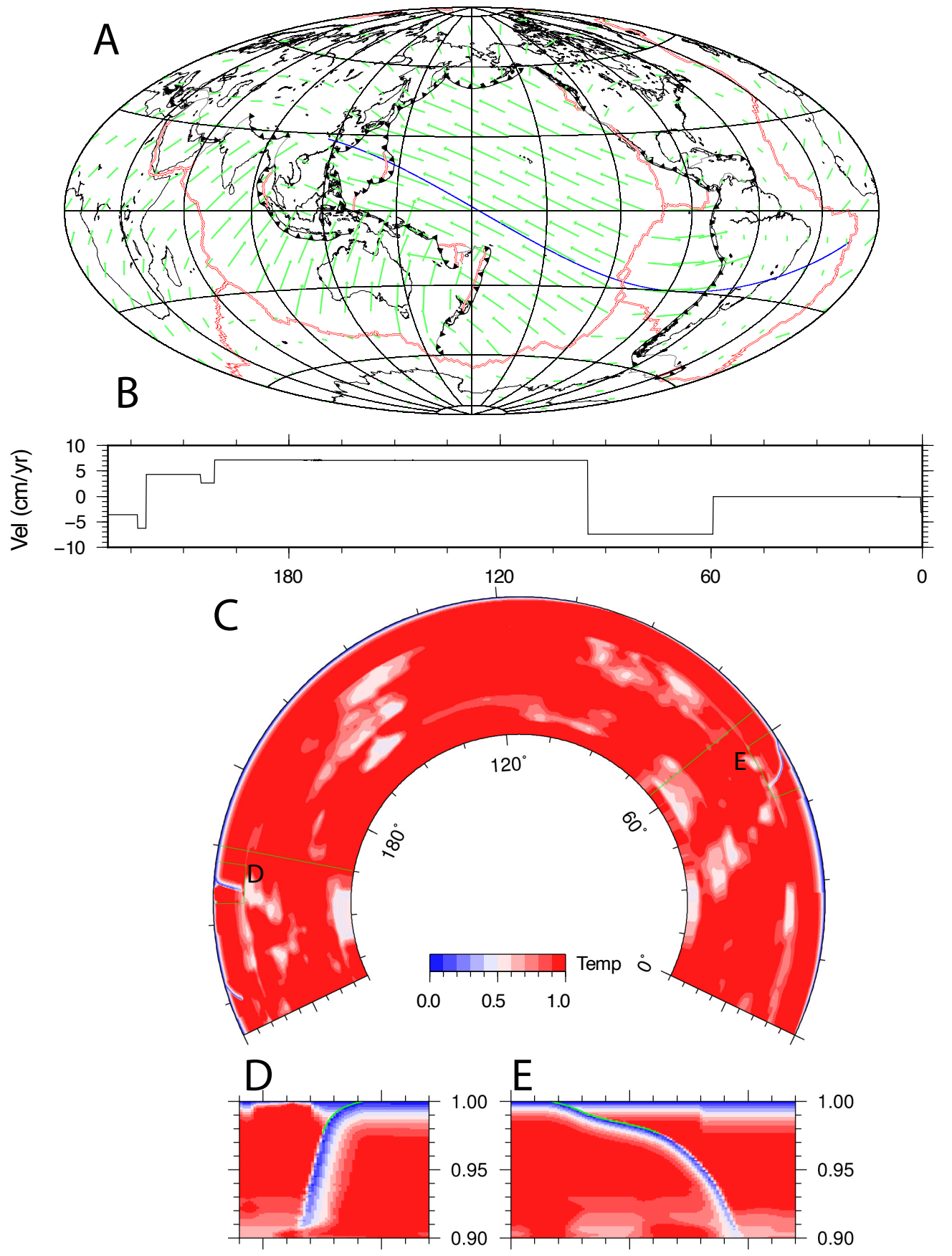

Figure 1. A. Velocity vectors in the no-net-rotation reference frame from MORVEL56 (Argus et al., 2011). Cross section indicated with blue line. B. Velocity in the direction of the cross section. C. Temperature distribution for cross section. Zoom-in of the Mariana (in D) and the Chilean (in E) slabs for the cross section. In $\mathrm{D}$ and $\mathrm{E}$, the solid green lines show the position of the weak zones.

frame was used since the sidewalls on two-dimensional cross sections preclude any large-scale differential motion between the bulk of the mantle and plates, that is, any net rotation. We account for uncertainty in plate motions with the standard deviation $\sigma_{\text {data }}$. 
Table 1. Assumed parameters that remain constant throughout the inference.

\begin{tabular}{ccc}
\hline Symbol & Parameter & Value \\
\hline$\rho$ & Density & $3300 \mathrm{~kg} / \mathrm{m}^{3}$ \\
$g$ & Gravity & $9.81 \mathrm{~m} / \mathrm{s}^{2}$ \\
$\alpha$ & Coefficient of Thermal expansion & $2 \times 10^{-5}$ \\
$\Delta T$ & Temperature Difference & $1400 \mathrm{~K}$ \\
$D$ & Depth of layer & $1500 \mathrm{~km}$ \\
$\kappa$ & Thermal Diffusivity & $10^{-6} \mathrm{~m} / \mathrm{s}$ \\
$\eta_{\text {ref }}$ & Reference Viscosity & $10^{20} \mathrm{~Pa}-\mathrm{s}$ \\
$\mathrm{Ra}$ & Rayleigh Number & $2.92 \times 10^{9}$ \\
$E_{a, \text { lo }}$ & Activation energy in lower mantle & $5.2 \times 10^{5} \mathrm{~J} / \mathrm{mol}$ \\
$1 / n_{\mathrm{lo}}$ & Strain rate weakening exponent in lower mantle & 1.0 \\
\hline
\end{tabular}

Selecting a set of representative cross sections in which all of the driving forces may be represented in two-dimensions is difficult, since no plate and subduction zone is likely to be truly two dimensional. Wide cross sections with plate motion parallel to a great circle orthogonal to a subduction zone are rare, and so we focused on a cross section in which plate motion was generally orthogonal to the trench. To investigate the mechanical coupling for subduction zones with various degrees of seismic coupling, we consider a cross section (Fig. 1) spanning about $240^{\circ}$ and containing three subduction zones that range from the seismically coupled (Chile) to the least coupled (Mariana). This cross section contains one subduction zone with back-arc extension near the Mariana trench. A weak zone factor line $\Gamma_{i}$ defined by the Slab 1.0 surface (Hayes et al., 2012), as defined in (6), was used. For all subsequent models, we assume the constant values of mantle parameters summarized in Table 1 .

When solving the nonlinear Stokes flow problem (1-2), it is crucial to resolve the thermal boundary layers and fault zones. This requires using either a discretization on a fine uniform mesh, which is computationally very expensive, or a discretization on an adaptively refinement mesh, which is algorithmically more complex. Here we use adaptive mesh refinement (AMR) to refine the mesh in areas such as oceanic plates, slabs, the mantle wedge, and fault zones. The resulting adaptive meshes exhibit a difference of eight refinement levels, which corresponds to a factor of 256 for the side lengths between the coarsest and finest mesh elements. We reach the finest resolutions at fault zones, where the spacing of degrees of freedom for velocity and pressure is only about $0.6 \mathrm{~km}-\mathrm{a}$ small fraction compared with the $25,644 \mathrm{~km}$ extension of the domain.

Our AMR capabilities are based on the p4est library (Burstedde et al., 2011, 2013), which manages adaptive meshes in parallel by using scalable algorithms that exploit topological "oc- 
tree" structures of the mesh. For the function approximation, we use quadratic finite elements for velocity and first-order discontinuous elements for pressure to ensure local mass conservation within each element. Resolving the fine-scale structures of the thermal boundary layers and fault zones through AMR is performed dynamically while solving the nonlinear Stokes equations; thus, the mesh is refined during the nonlinear solution process. The computational challenges of solving these nonlinear systems are severe. We use an advanced nonlinear solver based on Newton's method, which was specifically developed for viscoplastic Stokes flow problems (Rudi et al. 2020). The nonlinear solver is combined with a linear solver into an inexact Newton-Krylov method. The linearized Stokes systems arising at each Newton iteration are solved with a preconditioned Krylov GMRES method, where the preconditioning consists of an inverse Schur complement approximation (Rudi et al., 2017) and hybrid spectral-geometric-algebraic multigrid detailed in Rudi et al. (2015). This multigrid approach uses spectral coarsening of the polynomial order of finite elements, followed by a sequence of geometric multigrid levels coarsening the adaptive mesh, and concludes with algebraic multigrid. Code details and validation are provided in Rudi (2018).

\section{NUMERICAL RESULTS OF BAYESIAN INFERENCE}

We infer rheological parameters that best fit observed plate motions with a series of numerical experiments, which are summarized in Table 2. We determine how robust our algorithms for inference are by computing MAP estimates from different initial guesses of the stress exponent, $n$, and by how the parameter $n$ is treated. For these sets of experiments (A.1 and A.2 in Table 2), we observe multiple indicators that signify convergence to a stationary set of parameters: the reduction of the objective function (i.e., misfit between observed plate motions and model output), the decrease of the norm of the gradient of the inverse problem, and the decrease in step length from one iteration of the optimization algorithm (Newton BFGS) to the next (Fig. 2 and Supplementary Material). The eight parameters—nonlinear exponent, yield stress, activation energy, three coupling factors, and upper and lower mantle scaling factors-all converge to stable values in about twenty iterations (Supplementary Material). 
Table 2. Summary of numerical experiments and their purpose. Curly braces represent a set of experiments.

\begin{tabular}{clclc}
\hline $\begin{array}{c}\text { Inference } \\
\text { series }\end{array}$ & $\begin{array}{l}\text { Purpose } \\
\text { of experiments }\end{array}$ & $\begin{array}{c}\text { Velocity std. dev. } \\
\sigma_{\text {data }}[\mathrm{mm} / \mathrm{yr}]\end{array}$ & $\begin{array}{l}\text { Inference } \\
\text { parameter }\end{array}$ & $\begin{array}{c}\text { Initial guess } \\
n\end{array}$ \\
\hline A.1 1 & show convergence & 1.0 & $m_{n} \propto 1 / n$ & $\{2.0,2.8,3.2\}$ \\
A.2 & show convergence & varies by plate size & $m_{n} \propto 1 / n$ & $\{2.0,2.8,3.2\}$ \\
B.1 & show error dependence & $\{0.25,0.5,1.0,2.0,4.0\}$ & $m_{n} \propto 1 / n$ & 2.8 \\
B. 2 & show error dependence & $\{0.25,0.5,1.0,2.0,4.0\}$ & $m_{n} \propto n$ & 2.8 \\
C. 1 & show posterior details & varies by plate size & $m_{n} \propto 1 / n$ & 2.8 \\
\hline
\end{tabular}

We now look at the initial guess in the context of earlier work using synthetic data with known rheological parameters, with conditional and marginal distributions computed through repeated forward solves (without assumptions on their form using MCMC). In this work, Ratnaswamy et al. (2015) found mostly unimodal posterior distributions that were well approximated by Gaussian distributions near the best-fitting values (MAP points). For the Pacific cross-section data with two different configurations of data errors, discussed below, we estimate rheological parameters with three different guesses on $n(2.0,2.8$, and 3.2). In all cases, the optimization algorithm converges on the same $n \approx 2.8$ (Fig. 2A), indicating that there does not appear to be any substantial local minimum for cross-sectional inference with geophysical data. We observe that other parameters

A

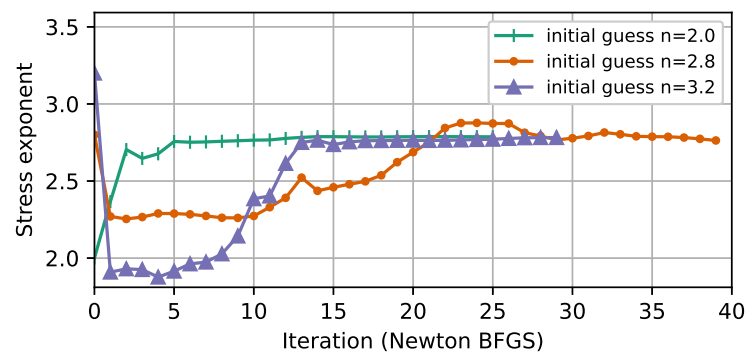

C

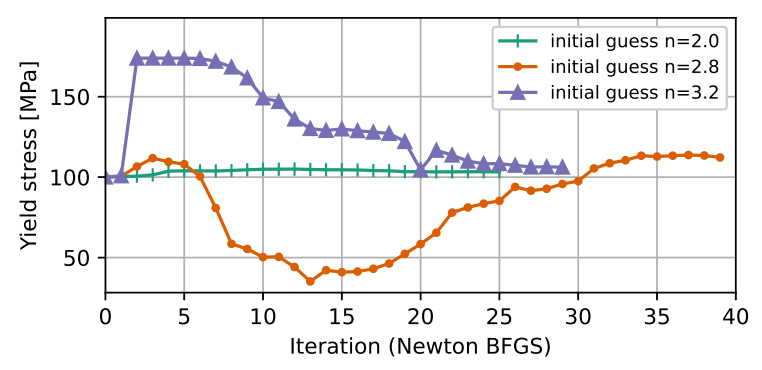

B

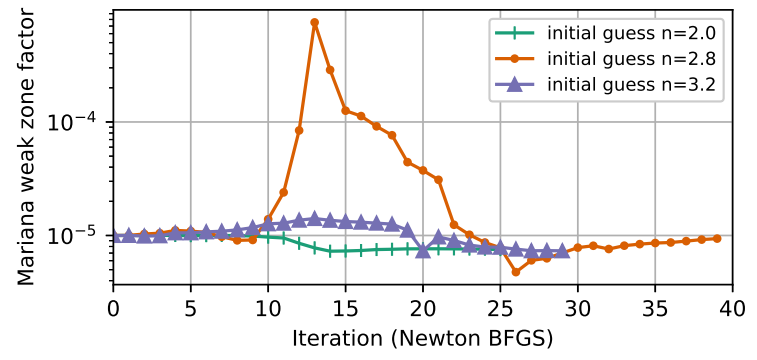

D

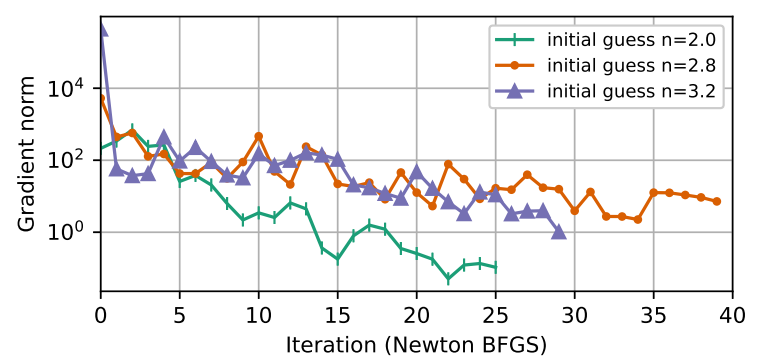

Figure 2. For series A.1, convergence of optimization algorithm (Newton BFGS) to find the optimally fitting parameters using different initial guesses for the stress exponent $n$. The different initial guesses are $n=2.0, n=2.8$, and $n=3.2$. The three (out of eight) parameters-stress exponent (A), Mariana weak zone factor (B), and yield stress (C) - converge to the same optimum values regardless of initial guesses. Correspondingly, the gradient norm of the inverse problem is reduced by a factor between $10^{-4}$ and $10^{-3}$. 
such as yield stress and weak zone factor also converge to the same respective values regardless of

Our focus on the stress exponent, $n$, comes from the strong sensitivity of the inverse problem with respect to $n$, which is further documented below. In addition to different initial guesses for $n$, we test the influence of the transfer function that maps a (normalized) inference parameter value $m_{n}$ to the value $n$ used in the Stokes model. One version of the transfer function is linear, $m_{n} \propto n$, and the other version is inverted, $m_{n} \propto 1 / n$. Hence $m_{n}$ directly represents the strain rate weakening exponent (up to a scaling factor). With these different choices of transfer functions, the resulting MAP points for all parameters are nearly the same (Supplementary Figs. S9 and S12). The only noticeable difference is the upper mantle (UM) scaling parameter when small data errors $\left(0.5 \mathrm{~mm} / \mathrm{yr}\right.$ or below) are assumed, in which case the parameter becomes smaller when $m_{n} \propto n$ (series B.2) compared with $m_{n} \propto 1 / n$ (series B.1). However, the reduction of UM scaling in series B.2 is accompanied by a small reduction in $n$ and a small increase in $\sigma_{y}$, both of which cause less weakening and thus must be compensated by a reduction in UM scaling. These changes are consistent with the variations evident within marginal distributions that we discuss below. In summary, the similarity of the resulting MAP points indicates that the choice of transfer function does not alter the recovered MAP, and the choice can be made based on modeling decisions for the prior and posterior distributions.

From inference series B.1 (where $m_{n} \propto 1 / n$ ) considering the case with the smallest assumed data error $\left(\sigma_{\text {data }}=0.25 \mathrm{~mm} / \mathrm{yr}\right.$ ), we have a model (Fig. $3 \mathrm{C}$ ) that fits the data the closest; the optimization method rapidly minimizes the cost function during several iterations with the forward 5 velocity converging toward the plate motion data, including the major plates and small plates in the back-arc (Fig. 3C). The effective viscosity through the entire domain varies between $10^{18}$ and $10^{24}$ Pa-s with major strain thinning within the upper mantle below the plates, in a halo above the slab in the upper mantle, and weakening in the hinge zone of each subducting plate (Fig. $3 \mathrm{C}$, right). In this inference, the viscosity structure of the Mariana subduction zone differs substantially from that of Chile. The Mariana shows more weakening of the slab throughout the hinge zone, below the imposed weak zone (e.g., the fault). There is also weakening above the fault on the overriding 
A
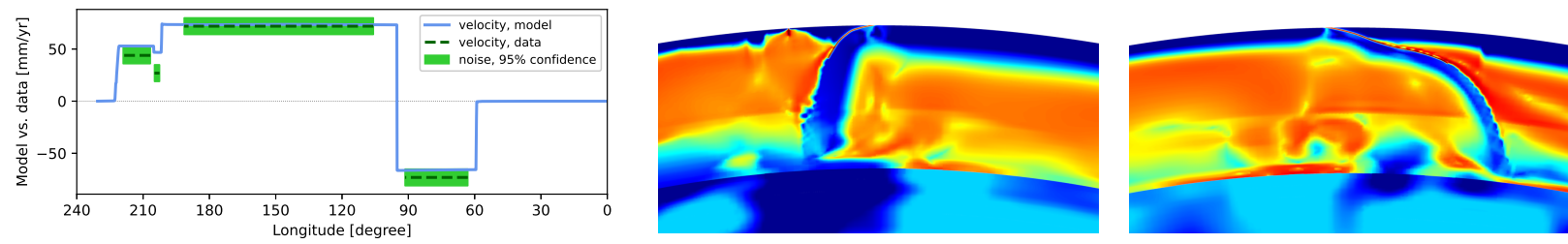

$\mathrm{B}$
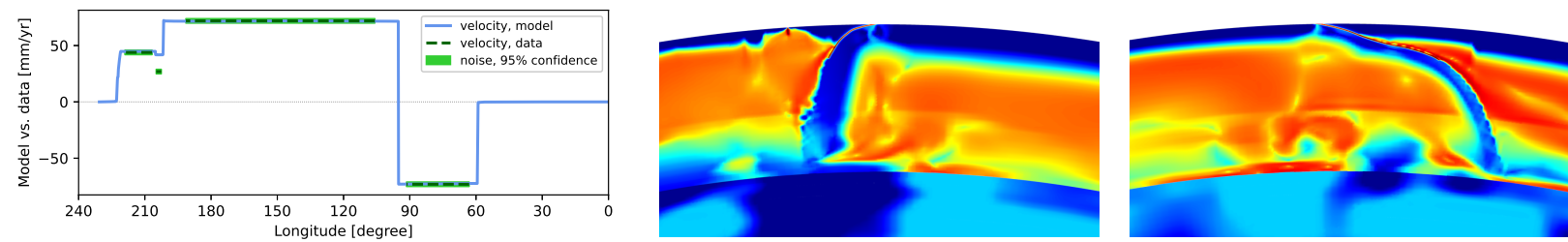

C

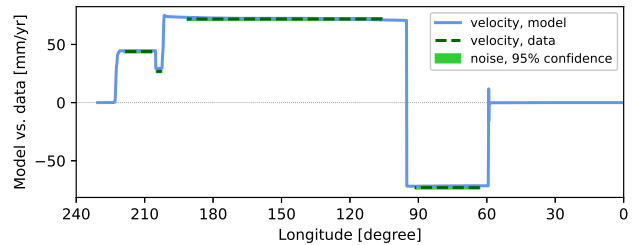

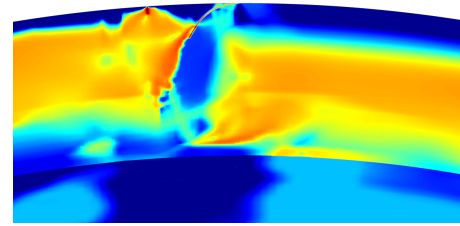

Mariana

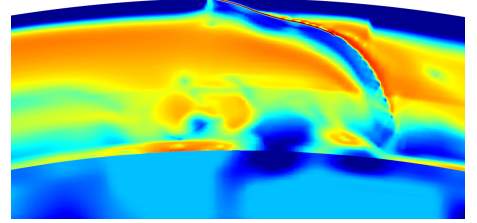

Chile

Figure 3. Results for series B.1. Velocities at the surface from model output after completion of inference, using decreasing standard deviations of the data error $\sigma \in\{0.5,1.0,4.0\}$ [mm/yr] (bottom to top). Effective viscosity around plate margins (center and right columns).

plate (Mariana microplate), but the weakness of the fault is only $10^{-4}$ compared with the value for Chile of $10^{-6}$, as we will see in more detail. In Chile, the weakening of the hinge zone is spatially limited and occurs only below where the fault intersects the surface.

The results depend on the magnitude and type of observation errors, that is, on $\sigma_{\text {data }}$, where $\sigma_{\text {data }}$ is assumed to contain data and model errors and it is incorporated in $\mathcal{C}_{\text {data }}$ from $(8)$. We model errors in two ways: (i) with a constant value for each plate and (ii) with an error that depends on plate size. For the first set of inverse problems, we vary the standard deviation of the data misfit term, $\sigma_{\text {data }} \in\{4,2,1,0.5,0.25\}[\mathrm{mm} / \mathrm{yr}]$. With a large value of $\sigma_{\text {data }} \geq 1 \mathrm{~mm} / \mathrm{yr}$, the estimate for the plate motion data for the larger plates, especially for the large, fast-moving Pacific Plate, generally fits the data well for any of the assumed data errors. But the small back-arc basin for the Mariana subduction zone is not well fit (Fig. 3B,C). When the data error is large, the surface plate motions do not display divergence above the Mariana slab. As $\sigma_{\text {data }}$ is reduced, the fit of the velocity of this small Mariana plate improves, especially between $\sigma_{\text {data }}$ values of 1 and $0.5 \mathrm{~mm} / \mathrm{yr}$. 
A

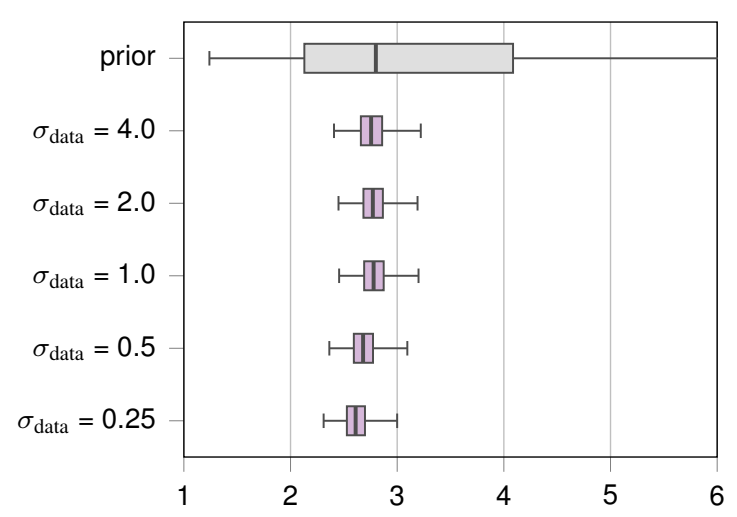

C

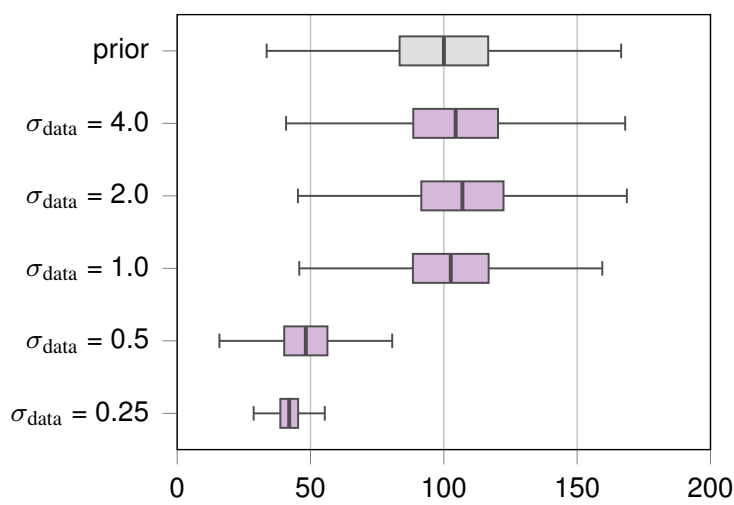

B

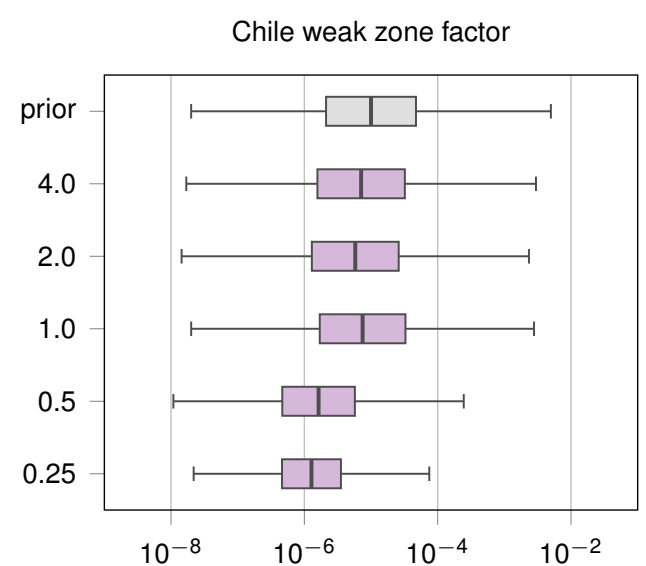

D

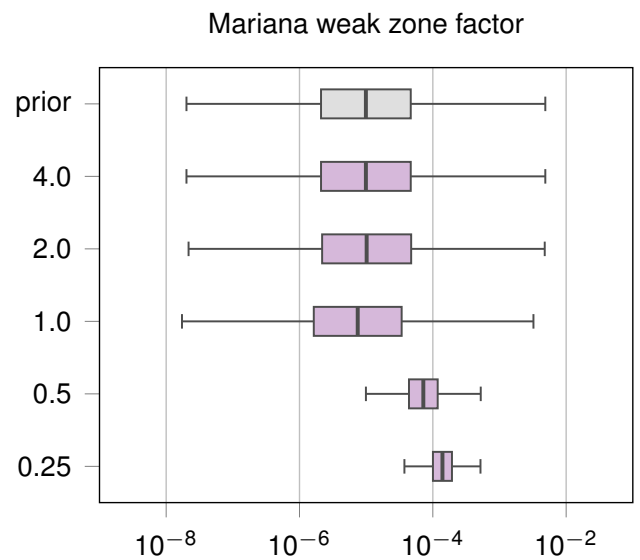

Figure 4. For series B.1. Prior and inferred posterior uncertainties for decreasing data misfit standard deviations for A. stress exponent, B. Chile weak zone factor, C. yield stress, and D. Mariana weak zone factor. Box represents interquartile range (IQR), from the first quartile (Q1)/25th percentile to the third quartile $(\mathrm{Q} 3) / 75$ th percentile, capturing $50 \%$ of the distribution; whiskers show the minimum $(\mathrm{Q} 1-1.5 \times \mathrm{IQR})$ and maximum $(\mathrm{Q} 3+1.5 \times \mathrm{IQR})$, and the bar inside the box shows the MAP estimate.

the recovered parameters (Fig. 4): The global yield stress drops from about $100 \mathrm{MPa}$ to about 45 $\mathrm{MPa}$, which leads to much more (e.g., broader scale) yielding within the hinge zones of the three slabs. When the yield stress decreases, there is a jump in the weak zone factor for the Mariana from $10^{-5}$ to $10^{-4}$ (Fig. $4 \mathrm{C}$ ). Essentially, the divergence above the slab, referred to as trench rollback, causes the Mariana slab to roll back. The slab is able to roll back only if it can easily bend in the hinge zone and a lower effective viscosity in the hinge zone is required (Alisic et al., 2012); consequently, fitting the roll-back in the kinematic data well leads to a global reduction in the yield stress and hence the quite evident sharp reduction of $\sigma_{y}$ as $\sigma_{\text {data }}$ decreases. While it is interesting that the model is capable of fitting the back-arc motion, the inferred parameters might be a result of under-estimating observation and model errors.

We now explore the second parameterization of $\sigma_{\text {data }}$ by making data errors proportional to 
Table 3. Inference series C.1. Prior and posterior modes and standard deviations. Standard deviations are "additiv" $( \pm)$ for normal distributions and "multiplicative" $(*)$ for log-normal distributions.

\begin{tabular}{lrcccl}
\hline Parameter & Distribution & \multicolumn{2}{c}{ Prior } & \multicolumn{2}{c}{ Posterior } \\
& & mode & std. dev. & mode & std. dev. \\
\hline Scaling in upper mantle & log-normal & $5.0 \times 10^{7}$ & $* 5.0$ & $4.3 \times 10^{7}$ & $* 5.0$ \\
Activation energy [J/mol] & normal & $5.2 \times 10^{5}$ & $\pm 5.8 \times 10^{4}$ & $5.3 \times 10^{5}$ & $\pm 6.8 \times 10^{4}$ \\
Scaling in lower mantle & log-normal & 90.0 & $* 5.0$ & 197.8 & $* 1.7$ \\
Stress exponent & inverse normal & 2.80 & $-0.89,+2.45$ & 2.78 & $-0.14,+0.16$ \\
Yield stress [MPa] & normal & 100.0 & \pm 24.6 & 94.3 & \pm 16.1 \\
Chile weak zone factor & log-normal & $1.0 \times 10^{-5}$ & $* 10$ & $7.9 \times 10^{-6}$ & $* 8.5$ \\
Mariana weak zone factor & log-normal & $1.0 \times 10^{-5}$ & $* 10$ & $4.9 \times 10^{-6}$ & $* 9.4$ \\
Ryuku weak zone factor & log-normal & $1.0 \times 10^{-4}$ & $* 10$ & $1.2 \times 10^{-3}$ & $* 2.1$ \\
\hline
\end{tabular}

plate size. Essentially, when the error is constant for each data value (recall we have one data value per node in the finite element mesh within the interior of plates), the larger plates are weighted more strongly, as we have already seen with the Pacific Plate being well fit even for large data errors. However, the small plates, although geodynamically of substantial importance, exert little control in the inference. We address this in inference series C.1 by assuming a data standard deviation proportional to the square root of the plate size. The inference with this plate sizedependent error leads to a good fit for most plates, from the large Pacific Plate to the Mariana microplate (Fig. 5). We provide more details of this inference series in Table 3 . The table lists the modes of prior and posterior for each parameter, where a mode of the posterior coincides with the MAP point. It further summarizes properties of the posterior by providing standard deviations, which are obtained by marginalizing the posterior covariance matrix. We can learn from this table that for the parameters, where standard deviations in the posterior are reduced relative to the prior, the data and model are able to inform these parameters.

Looking at estimated parameters with plate size-dependent data errors in more detail, we see that a number of parameters show a positive trade-off with the stress exponent, $n$, evident through 2-D marginal distributions (Fig. 6 and Fig. S18 in the Supplementary Material). Both the yield stress and the stress exponent control the nonlinearity of the material, and a high $n$, which leads to more weakening, must trade off with a higher $\sigma_{y}$ so that there is less volume (usually in the hinge zone) that experiences yielding. The activation energy, $E_{a}$ (which is the dimensional quantity associated with $\beta$ in (5)), trades off with $n$, since a high $E_{a}$ by itself leads to higher viscosities within the cooler parts of the domain (the slabs and plates). Therefore, a larger $n$ is needed to 
A
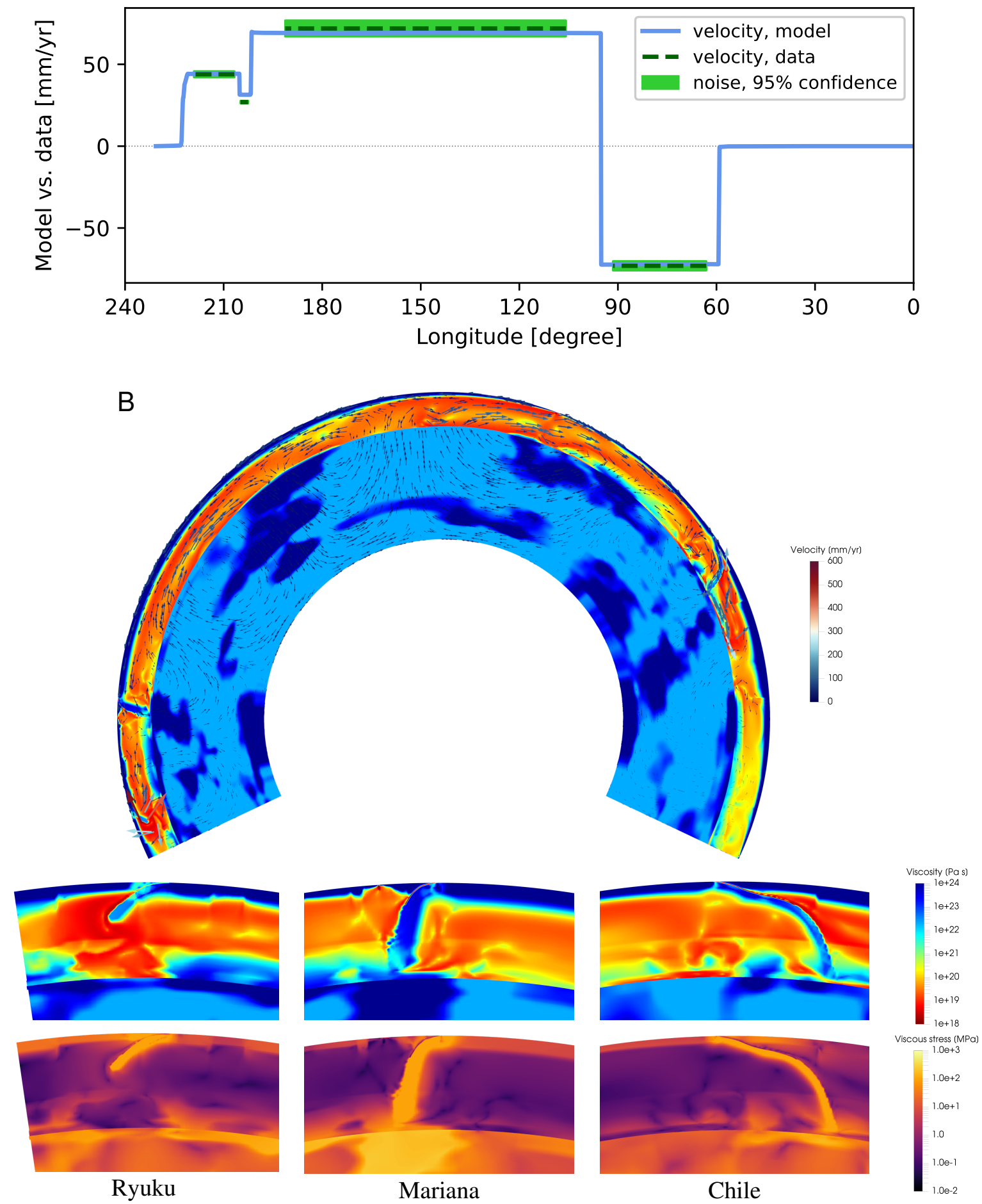

C

Figure 5. For series C.1 A. Tangential velocities along top surface. B. Effective viscosities for whole domain overlain by velocity vectors for converged solution. High-resolution image of effective viscosity (C) and square root of second invariant of stress (D) for three subduction zones.

weaken this cold material. There is also a strong trade-off of $E_{a}$ with $\sigma_{y}$ (Supplementary Fig. S18). This trade-off between $n$ and $E_{a}$ has been suggested in early studies of the lithosphere and mantle using forward models (Christensen, 1983). 
Table 4. Averages of fields in C.1

\begin{tabular}{lllll}
\hline Field & Upper mantle & Chile & Mariana & Ryuku \\
\hline Effective viscosity (Pa-s) & $6.15 \times 10^{19}$ & $1.54 \times 10^{23}$ & $1.81 \times 10^{23}$ & $2.67 \times 10^{23}$ \\
$\langle\langle\eta\rangle\rangle(\mathrm{Pa}-\mathrm{s})$ & $1.96 \times 10^{19}$ & $4.04 \times 10^{22}$ & $5.01 \times 10^{22}$ & $2.61 \times 10^{22}$ \\
Strain rate $\left(\mathrm{s}^{-1}\right)$ & $6.90 \times 10^{-15}$ & $1.00 \times 10^{-15}$ & $8.72 \times 10^{-16}$ & $1.64 \times 10^{-15}$ \\
Viscous stress (Pa) & $2.71 \times 10^{5}$ & $8.08 \times 10^{7}$ & $8.74 \times 10^{7}$ & $8.55 \times 10^{7}$ \\
\hline
\end{tabular}

We further consider the inference series C.1 with plate size-dependent error, and we take the procedure a step further by inferring the normal and tangential (shear) stresses within the fault zone. We find that the weak zone factors for the Chile, Mariana, and Ryuku subduction zones vary from $1.9 \times 10^{-6}$ to $1.2 \times 10^{-3}$ (Table 4) - a substantial variation over nearly three orders of magnitude. However, we observe that the average tangential and normal stresses for these faults varies by a much smaller degree (Fig. 7). The values for the normal stresses vary between 83 and 110 $\mathrm{MPa}$ while the tangential stresses vary between 3 and $22 \mathrm{MPa}$. This interplay between rheological parameters is important and demonstrates the nonlinearity and strong interactions between global rheological parameters (yield stress and strain rate exponent) and local coupling parameters.

\section{DISCUSSION}

We have inferred rheological parameters (nonlinear exponent, yield stress, and activation energy), viscosity prefactors within the upper mantle and hinge zone of the subducting plate, the coupling coefficient and stresses within several subduction megathrusts, and the covariances between them. Since inference is carried out in a two-dimensional geometry where it is difficult to isolate plate forces and kinematics solely along a cross section, the results are preliminary and illustrative of the potential of the approach. The method overcomes substantial limitations in previous geodynamic inference by not needing to simplify the rheogical laws. Moreover, when placed in the perspective of prior work with synthetic data constructed with forward models showing recovery of known nonlinear rheological parameters (Ratnaswamy et al., 2015), the results point to a considerable robustness in this application of adjoint-based MAP estimation in plate and mantle dynamics. However, the inferred rheological parameters and plate coupling values stem from a single (universal) form governed by stress and temperature. This means that other processes that could influence deformation in the lithosphere and mantle, such as grain size and volatile concentrations, might 
A

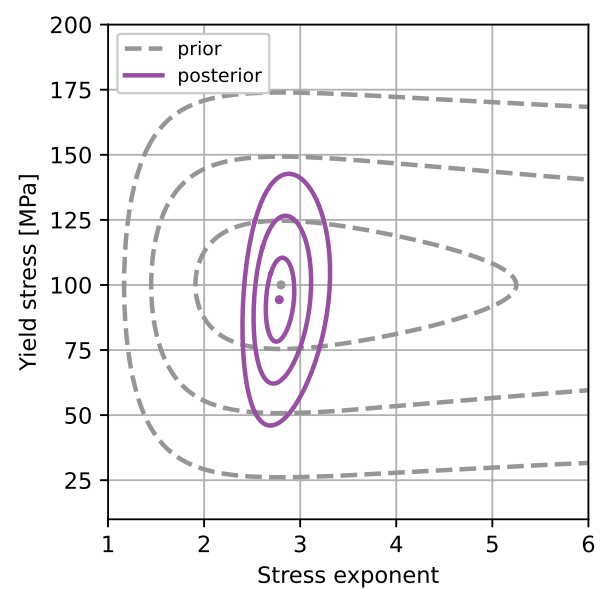

C

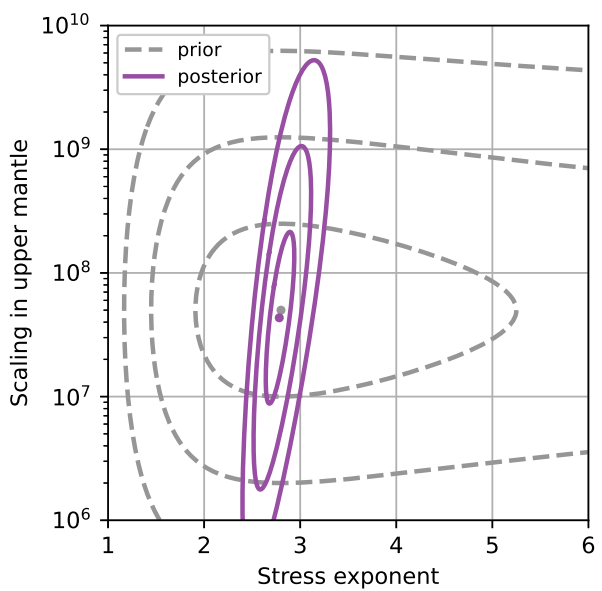

B

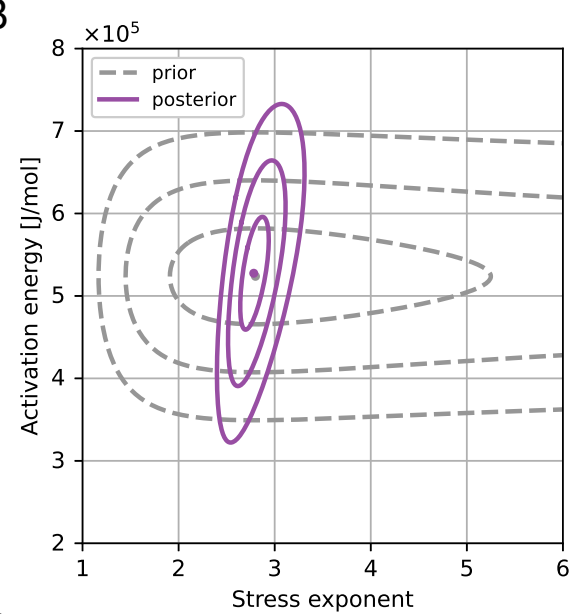

D

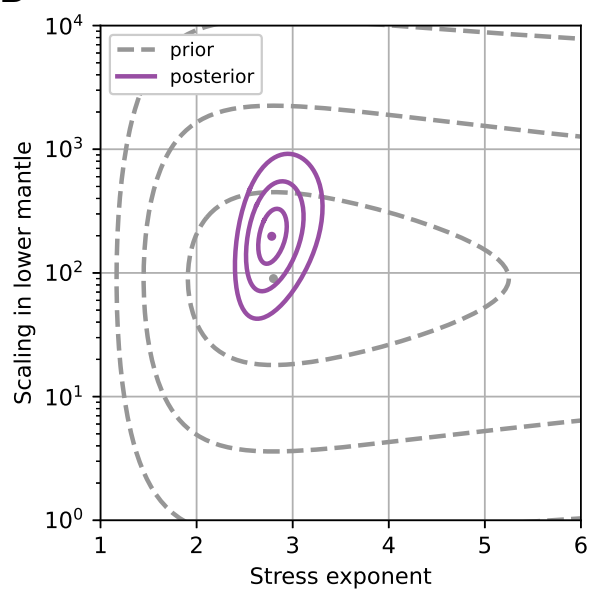

Figure 6. Posterior (purple) and prior (gray) 2-D marginals from series C.1. A. Yield stress versus stress exponent, B. activation energy, C. scaling in the upper mantle, and D. scaling in the lower mantle all versus the stress exponent. The contour intervals are 1,2, and 3 standard deviations.

A

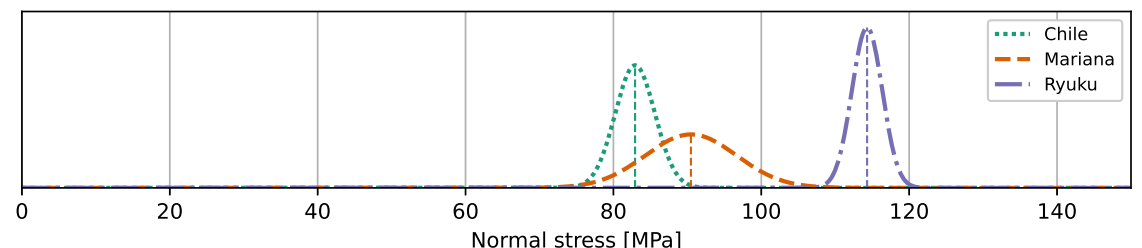

B

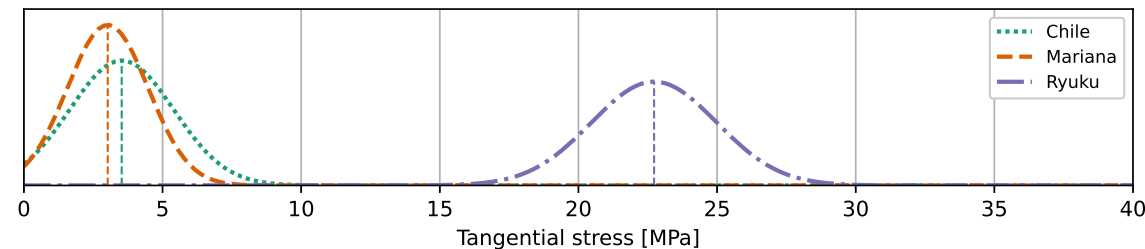

Figure 7. For series C.1, components of the stress along the weak zone surfaces: A. normal stresses; B. tangential stresses. 
be mapped together. Grain size and volatiles could vary spatially in the mantle (Riedel \& Karato, 1997; Hirth \& Kolhstedt, 1996; Spasojevic et al., 2010), and these would likely lead to a spatial „variation in the dominant deformation mechanism in different parts of the mantle (Hall \& Parmentier, 2003). An alternative approach would require that the rheological parameters (like the nonlinear exponent, $n$ ) vary spatially. Exploration of this method with synthetic data showed that with plate motion constraints and with many more unknowns than independent data, this inference method was less effective at parameter recovery while showing rapid decay in parameter recovery with depth (Worthen et al. 2014). A compromise could be a tectonic regionalization to the rheological parameters, such as a small set of nonlinear exponents, $n$, that vary with tectonic type (e.g., within the mantle wedge versus below cratons). This was attempted successfully with a Markov chain Monte Carlo approach by Baumann et al.(2014) in a two-plate system with different parameters for the plates and the crust versus the mantle. Considerable opportunities remain for future exploration in this direction using the adjoint method once full global inference become available.

We consider the results that show similarities and differences from prior experimental work and geophysical models. The inferred strain rate exponent, $n$, is $2.8 \pm 0.2$ (i.e., mean value plus/minus one standard deviation), using nonrestrictive (permissive) priors. Considerable research on the creep of olivine suggests that, under a dislocation regime, wet olivine could have $n=3.0$ Karato \& Wu, 1993), $3.5 \pm 0.3$ (Hirth \& Kohlstedt, 2003), or $3.6 \pm 0.24$ (Korenaga \& Karato, 2008). The ¿values for dry olivine are even higher, with $n=3.5$ (Karato \& Wu, 1993), $3.5 \pm 0.3$ (Hirth \& Kohlstedt, 2003), or 4.94 \pm 0.05 (Korenaga \& Karato, 2008). The MAP estimates from our models are smaller than these experimental values but overlap at the $95 \%$ confidence level.

${ }_{435}^{4} \quad$ Mantle and lithosphere deformation becomes qualitatively more nonlinear when $n>3$ (Alisic et al. 2012) since there is substantially more shear thinning in the upper mantle using experimental values compared with the recovered values. Earlier, using a global geometry but with a forward approach similar to the present inversions with resolved weak (shear) zones for the interplate faults and resolved hinge zones, Alisic et al. (2012) preferred an exponent, $n$, of about 3.0 and ${ }_{440}$ yield stress, $\sigma_{y}$, of about $100 \mathrm{MPa}$ when the models were fit to both the major plates and the microplates adjacent to trenches that rapidly roll back. Although these global models suggest a 
larger $n$ and about the same yield stress from what we recover from the cross-sectional inference, they are within the $95 \%$ confidence interval of the 2-D marginal distributions (Fig. 6). We currently cannot determine whether this difference between the two sets of models is due to the geometry (2-D cross-sectional versus full spherical), the more realistic parameterization of the fault zones in this inference, or some other reason. Speculating that the change in fault parameterization causes the differences is not unreasonable: The Alisic et al.(2012) study had near-surface dip angles for the faults that were steeper, and fits to the plate motions could have been accomplished through more strain rate weakening achieved by a higher $n$ than otherwise inferred with more realistic, continuously curving fault zones.

Taking the geophysical models, either the spherical or the cross-sectional, with $n=3.0$ or 2.8 at face value suggests that the nonlinear exponent may not be as high as preferred from experimental work for either dry olivine (3.0 to 3.6) or wet olivine (3.5 to 4.9). Since we impose a non-negativity restriction (Tarantola, 2005) on the inferred parameters, strain rate exponents less than zero cannot be recovered, and so the hypothesis for a velocity-strengthing material cannot be precluded. Bercovici (1993) found that a value of $n=-1$ can produce plate-like behavior, but without yielding or temperature-dependence, both included here. The inferred activation energies are $530 \pm 16 \mathrm{~kJ} / \mathrm{mol}$, which is in the range of dry olivine, $540 \mathrm{~kJ} / \mathrm{mol}$, but more than the expected values for wet olivine, $430 \mathrm{~kJ} / \mathrm{mol}$, respectively, in the dislocation creep regime (Karato \& Wu. 1993). We see a strong trade-off with $n$.

We find yield stresses of $94 \pm 16 \mathrm{MPa}$, six times smaller than those found in rock mechanics experiments (600 MPa) (Mei et al., 2010), a conclusion in line with previous studies using forward geodynamic models with generic plate motions (Zhong \& Gurnis, 1996; Moresi \& Gurnis, 1996). However, this postiori distribution is not significantly different from the prior. The mean of 94 MPa is slightly smaller than the preferred values found in the global forward models of Alisic et al. (2012), where $100 \mathrm{MPa}$ was preferred. The current approach of using seismic constraints from Slab 1.0 (Hayes et al., 2012), as just discussed, allows subducting plates to continuously bend within the hinge zone, as the earlier models used a steeper dip angle to define the weak 
zones. The moderately higher yield stresses found here will recover more realistic estimates once we can achieve full spherical models.

We find an average (arithmetic mean) viscosity of the upper mantle through the entire cross section to be $6 \times 10^{19} \mathrm{~Pa}$-s. The average samples the upper mantle from the base of the thermal lithosphere to $410 \mathrm{~km}$ depth, while excluding the thermal slab, and is dominated by the suboceanic environment. We note that the Pacific and Nazca Plates several hundred kilometers from the Mariana and Chile trenches are about $10^{19} \mathrm{~Pa}$-s, at the higher end of the $5 \times 10^{17}$ to $1 \times 10^{19} \mathrm{~Pa}$-s inferred for the Indian Plate several hundred kilometers from the Sumatra Trench using transients excited by the 2012 Indian Plate earthquake (Hu et al., 2016). In global models, it will be possible to alter the adjoint-based inference approach to incorporate priors within specific regions in the lithosphere and upper mantle where we have explicit geodetic constraints from transients induced by co-seismic, lake, and glacial loads.

For thermal convection with variable viscosity, Christensen (1984) has argued that a strain rateweighted viscosity, which we call $\langle\langle\eta\rangle\rangle$, is more appropriate since what limits convection is the viscosity in those regions undergoing the strongest deformations. The strain rate-weighted average viscosities within the Chile, Mariana, and Ryuku hinge zones are 4.0, 5.0, and $2.6 \times 10^{22} \mathrm{~Pa}$-s, respectively, all about the same as the $6 \times 10^{22} \mathrm{~Pa}-\mathrm{s}$ inferred from simple bending models of linearviscous plates (Buffett \& Rowley, 2006). Whether $\langle\langle\eta\rangle\rangle$ is more appropriate for the asthenosphere is not entirely certain, but it is the most sensible for the hinge zone where the forces transmitted to the subducting plates are strongly influenced by resistance from the bending plate that yields while it bends.

High resolutions of the computational mesh are required to resolve the weak zones (faults), which are characterized by an intrinsic material property (e.g., the coupling factors). Additionally, the hinge zones, where the bending plates yield and drive rapidly moving microplates, also need to be resolved. Hence, high-resolution forward models are essential for meaningful inference results. In addition, the parameterization of data errors can strongly influence the proper inference of small-scale features such as back-arcs, fault zones, and bending plates. The computational experiments with different data errors allow us to demonstrate how the material properties change 
between cases where the microplates sufficiently contribute to the objective function of the inverse problem versus when they have relatively small weights compared with larger plates. A small constant data error can lead to overfitting. This motivated us to vary data error according to the square root of plate size, allowing us to mitigate over fitting and achieve the back-arc motion. The global yield stress can change substantially when such back-arc extension (trench roll-back) is resolved. How this will influence parameter estimation with a global, fully spherical geometry awaits to be demonstrated.

The coupling factors between the subducting and overriding plates, $w_{i}$, along with the associated stresses have been inferred. The coupling coefficients are intrinsic parameters whereas stresses are extrinsic. We find that two weak zones (Mariana and Chile) have small values of about $10^{-5}$ with large variances (Supplementary Material) while the Ryuku weak zone is substantially larger at about $10^{-3}$ with a smaller variance. This statement is independent of the yield stress and the nonlinear exponent, inspite of the large posteriori variance on both quantities. Both yielding and shear thinning can weaken the boundary between the plates, but the inference algorithm is finding coupling factors that vary by nearly two orders of magnitude. However, the effective viscosities and stresses within the shear zones do not vary by nearly this amount (Fig. 7). Although the total variation of the stresses in the upper mantle is about $10^{3}$ (from $\sim 0.1 \mathrm{MPa}$ to $\sim 200$ $\mathrm{MPa}$ ), the variation within similar dynamic elements (e.g., plate interfaces, plate interiors, slabs, and the central parts of the asthenosphere) are all much smaller (Fig. 5D). This is significant for understanding the occurrences of great earthquakes and other tectonic processes because despite substantial differences in the material properties of megathrust (such as differences in sediment thickness between trenches (Ruff, 1989; Heuret et al., 2011)) or in the strength of potential regional tectonic controls (such as plate age and convergence rates (Ruff \& Kanamori, 1980) $)$, the plate-mantle system will deliver nearly constant stresses to similar tectonic elements because of the strong nonlinearity in the rheology of the system (through both $n$ and $\sigma_{y}$ ).

Caution must be exercised in interpreting the geophysical significance of the difference between the large values for Ryuku and the small values for Mariana and Chile because of the two-dimensional nature of the forward model. The Ryuku slab only partially penetrates through 
the upper mantle in this particular cross section, and thus great resistance is required to slow down the Philippine Sea Plate (especially since we placed no constraint on the velocity of the Eurasian Plate, Fig. 57. The inferred shear stresses within the weak zones are consistently smaller than those of the normal stresses, consistent with models of the seismogenic process that usually start with the premise of a frictional material with shear stresses being a fraction of the normal stress (Scholz. 1990).

The inference results illustrate the substantial trade-offs expected between the global rheological and local coupling parameters and point to the strength of the approach. The adjoint-based inference method described here has been implemented in a finite element code that scales to millions of cores on parallel supercomputers (Rudi et al., 2015). It has been used extensively in ultra-high-resolution full spherical models for the present day (Stadler et al., 2010) and the geological past (Hu et al. 2021). This use suggests that such high-resolution inference of the highly nonlinear plate-mantle system are within reach.

\section{ACKNOWLEDGMENTS}

This work was partially supported by the U.S. National Science Foundation (NSF) through awards EAR-1645775 and EAR-2009935 to MG and EAR-1646337 and DMS-1723211 to GS. This material also was based in part upon work supported by the U.S. Department of Energy, Office of Science, under contract DE-AC02-06CH11357. Computations carried out on the Texas Advanced Computing Center (TACC) Stampede-2 supercomputer using allocation TG-DPP130002 of the Extreme Science and Engineering Discovery Environment (XSEDE) supported by NSF grant ACI1548562.

\section{DATA AVAILABILITY}

The authors declare that all other data supporting the findings of this study are available within the paper and its Supplementary Material files. The code (Rhea) used for the forward models and the inference is available upon request. 


\section{References}

Alisic, L., Gurnis, M., Stadler, G., Burstedde, C., Wilcox, L. C., \& Ghattas, O., 2010. Slab stress and strain rate as constraints on global mantle flow, Geophysical Research Letters, 37(22).

Alisic, L., Gurnis, M., Stadler, G., Burstedde, C., \& Ghattas, O., 2012. Multi-scale dynamics and rheology of mantle flow with plates, Journal of Geophysical Research: Solid Earth, 117(B10).

Argus, D. F., Gordon, R. G., \& DeMets, C., 2011. Geologically current motion of 56 plates relative to the no-net-rotation reference frame, Geochemistry, Geophysics, Geosystems, 12(11). Baumann, T. S., Kaus, B. J. P., \& Popov, A. A., 2014. Constraining effective rheology through parallel joint geodynamic inversion, Tectonophysics, 631, 197-211.

Bercovici, D., 1993. A simple model of plate generation from mantle flow, Geophysical Journal International, 114(3), 635-650.

Billen, M., 2008. Modeling the dynamics of subducting slabs, Ann. Rev. Earth Planet. Sci., 36, $325-356$.

Billen, M. I. \& Hirth, G., 2007. Rheologic controls on slab dynamics, Geochemistry, Geophysics, Geosystems, 8(8).

Borzì, A. \& Schulz, V., 2012. Computational Optimization of Systems Governed by Partial Differential Equations, SIAM.

Buffett, B., 2006. Plate force due to bending at subduction zones, J. Geophys. Res., 111.

Buffett, B. A. \& Rowley, D. B., 2006. Plate bending at subduction zones: Consequences for the direction of plate motions, Earth and Planetary Science Letters, 245(1), 359-364.

Burkett, E. \& Billen, M., 2009. Dynamics and implications of slab detachment due to ridgetrench collision, J. Geophys. Res., 114, 1-29.

Burstedde, C., Wilcox, L. C., \& Ghattas, O., 2011. p4est: Scalable algorithms for parallel adaptive mesh refinement on forests of octrees, SIAM Journal on Scientific Computing, 33(3), 11031133.

Burstedde, C., Stadler, G., Alisic, L., Wilcox, L. C., Tan, E., Gurnis, M., \& Ghattas, O., 2013. Large-scale adaptive mantle convection simulation, Geophysical Journal International, 192(3), 889-906. 
Christensen, U., 1983. Convection in a variable-viscosity fluid: Newtonian versus power-law rheology, Earth and Planetary Science Letters, 64(1), 153-162.

Christensen, U., 1984. Convection with pressure- and temperature-dependent non-Newtonian rheology, Geophysical Journal International, 77(2), 343-384.

Conrad, C. P. \& Lithgow-Bertelloni, C., 2002. How mantle slabs drive plate tectonics, Science, 298(5591), 207-209.

Crameri, F., Tackley, P., Meilick, I., Gerya, T., \& Kaus, B., 2012. A free plate surface and weak oceanic crust produce single-sided subduction on earth, Geophys. Res. Lett., 39(L03306).

Frank-Kameneëtìskiæi, D., 1969. Diffusion and heat transfer in chemical kinetics, vol. 2, Plenum Press (New York).

Garel, F., Goes, S., Davies, D., Davies, J., Kramer, S., \& Wilson, C., 2014. Interaction of subducted slabs with the mantle transition-zone: A regime diagram from 2-D thermo-mechanical models with a mobile trench and an overriding plate, Geochem. Geophys. Geosyst., 15, 1-27.

Gerya, T., 2011. Future directions in subduction modeling, J. Geodyn., 52, 344-378.

Gérault, M., Husson, L., M. S. Miller, M., \& Humphreys, E., 2015. Flat-slab subduction, topography, and mantle dynamics in southwestern Mexico, Tectonics, 34, 1892-1909.

Hall, C. \& Parmentier, E., 2003. Influence of grain size evolution on convective instability, Geochem. Geophys. Geosyst., 4, 1-27.

Hayes, G. P., Wald, D. J., \& Johnson, R. L., 2012. Slab 1.0: A three-dimensional model of global subduction zone geometries, Journal of Geophysical Research: Solid Earth, 117(B01302).

Heuret, A., Lallemand, S., Funiciello, F., Piromallo, C., \& Faccenna, C., 2011. Physical characteristics of subduction interface type seismogenic zones revisited, Geochemistry, Geophysics, Geosystems, 12(1).

Hinze, M., Pinnau, R., Ulbrich, M., \& Ulbrich, S., 2009. Optimization with PDE Constraints, vol. 23 of Mathematical Modelling: Theory and Applications, Springer Netherlands.

Hirth, G. \& Kohlstedt, D., 2003. Rheology of the upper mantle and the mantle wedge: A view from the experimentalists, in Inside the Subduction Factory, pp. 83-105, ed. Eiler, J., American Geophysical Union, Washington, DC. 
Hirth, G. \& Kolhstedt, D., 1996. Water in the oceanic upper mantle: implications for rheology, melt extration and the evolution of the lithosphere, Earth Planet. Sci. Lett., 144, 93-108.

Hu, J., Gurnis, M., Rudi, J., Stadler, G., \& Müller, D., 2021. Dynamics of abrupt change in Pacific Plate motion around $50 \mathrm{Ma}$, Nature Geoscience, in press.

Hu, Y., Bürgmann, R., Banerjee, P., Feng, L., Hill, E. M., Ito, T., Tabei, T., \& Wang, K., 2016. Asthenosphere rheology inferred from observations of the 2012 indian ocean earthquake, $\mathrm{Na}$ ture.

Kaipio, J. \& Somersalo, E., 2005. Statistical and Computational Inverse Problems, vol. 160 of Applied Mathematical Sciences, Springer-Verlag, New York.

Karato, S. \& Wu, P., 1993. Rheology of the upper mantle: A synthesis, Science, 260(5109), $771-778$.

Korenaga, J. \& Karato, S.-I., 2008. A new analysis of experimental data on olivine rheology, Journal of Geophysical Research: Solid Earth, 113(B2).

Mei, S., Suzuki, A., Kohlstedt, D., Dixon, N., \& Durham, W., 2010. Experimental constraints on the strength of the lithospheric mantle, Journal of Geophysical Research: Solid Earth, 115(B8).

Moresi, L. \& Gurnis, M., 1996. Constraints on the lateral strength of slabs from threedimensional dynamic flow models, Earth and Planetary Science Letters, 138(1-4), 15 - 28.

Müller, R. D., Roest, W. R., Royer, J.-Y., Gahagan, L. M., \& Sclater, J. G., 1997. Digital isochrons of the world's ocean floor, Journal of Geophysical Research: Solid Earth, 102(B2), 3211-3214.

Ranalli, G., 1995. Rheology of the Earth, Springer Science \& Business Media.

Ratnaswamy, V., Stadler, G., \& Gurnis, M., 2015. Adjoint-based estimation of plate coupling in a non-linear mantle flow model: theory and examples, Geophysical Journal International, 202(2), 768-786.

Riedel, M. \& Karato, S., 1997. Grain-size evolution in subducted oceanic lithosphere associated with the olivine-spinel transformation and its effects on rheology, Earth Planet. Sci. Letts., 148, 27-43.

Rudi, J., 2018. Global Convection in Earth's Mantle: Advanced Numerical Methods and Extreme-Scale Simulations, Ph.D. thesis, University of Texas at Austin. 
Rudi, J., Malossi, A. C. I., Isaac, T., Stadler, G., Gurnis, M., Staar, P. W., Ineichen, Y., Bekas, C.,

Curioni, A., \& Ghattas, O., 2015. An extreme-scale implicit solver for complex PDEs: highly heterogeneous flow in Earth's mantle, in SC15: Proceedings of the International Conference for High Performance Computing, Networking, Storage and Analysis, pp. 5:1-5:12, ACM.

Rudi, J., Stadler, G., \& Ghattas, O., 2017. Weighted BFBT preconditioner for Stokes flow problems with highly heterogeneous viscosity, SIAM Journal on Scientific Computing, 39(5), S272S297.

Rudi, J., Shih, Y.-h., \& Stadler, G., 2020. Advanced Newton methods for geodynamical models of Stokes flow with viscoplastic rheologies, Geochemistry, Geophysics, Geosystems, 21(9).

Ruff, L., 1989. Do trench sediments affect great earthquake ocurrence in subduction zones, PAGEOPH, 129, 263-282.

Ruff, L. \& Kanamori, H., 1980. Seismicity and the subduction process, Physics of the Earth and Planetary Interiors, 23(3), 240-252.

Scholz, C., 1990. The Mechanics of Earthquakes and Faulting, Cambridge University Press.

Scholz, C. \& Campos, J., 2012. The seismic coupling of subduction zones revisited, Journal of Geophysical Research: Solid Earth (1978-2012), 117(B5).

Simmons, N. A., Myers, S. C., Johannesson, G., \& Matzel, E., 2012. Llnl-g3dv3: Global p wave tomography model for improved regional and teleseismic travel time prediction, Journal of Geophysical Research: Solid Earth, 117(B10).

Spasojevic, S., Gurnis, M., \& Sutherland, R., 2010. Mantle upwellings above slab graveyards linked to the global geoid lows, Nature Geosci., 3, 435-438.

Stadler, G., Gurnis, M., Burstedde, C., Wilcox, L. C., Alisic, L., \& Ghattas, O., 2010. The dynamics of plate tectonics and mantle flow: From local to global scales, Science, 329(5995), $1033-1038$.

Tarantola, A., 2005. Inverse Problem Theory and Methods for Model Parameter Estimation, SIAM, Philadelphia, PA.

Tröltzsch, F., 2010. Optimal Control of Partial Differential Equations: Theory, Methods and Applications, vol. 112 of Graduate Studies in Mathematics, American Mathematical Society. 
Worthen, J., Stadler, G., Petra, N., Gurnis, M., \& Ghattas, O., 2014. Towards an adjoint-based inversion for rheological parameters in nonlinear viscous mantle flow, Physics of Earth and Planetary Interiors, 234, 23-34.

Zhong, S. \& Gurnis, M., 1995. Mantle convection with plates and mobile, faulted plate margins, Science, 267(5199), 838-843.

Zhong, S. \& Gurnis, M., 1996. Interaction of weak faults and non-newtonian rheology produces plate tectonics in a 3d model of mantle flow, Nature, 383(6597), 245.

Zhong, S., Gurnis, M., \& Moresi, L., 1998. Role of faults, nonlinear rheology, and viscosity 670 structure in generating plates from instantaneous mantle flow models, Journal of Geophysical Research: Solid Earth, 103(B7), 15255-15268. 
Government License: The submitted manuscript has been created by UChicago Argonne, LLC, Operator of Argonne National Laboratory ("Argonne”). Argonne, a U.S. Department of Energy Office of Science laboratory, is operated under Contract No. DE-AC02-06CH11347. The U.S. Government retains for itself, and others acting on its behalf, a paid-up nonexclusive, irrevocable worldwide license in said article to reproduce, prepare derivative works, distribute copies to the public, and perform publicly and display publicly, by or on behalf of the Government. The Department of Energy will provide public access to these results of federally sponsored research in accordance with the DOE Public Access Plan. http://energy.gov/downloads/doe-public-access-plan 